\title{
FÁBRICA DE ENSINO EM INDÚSTRIA 4.0: PROPOSIÇÃO DE MODELO DE NEGÓCIOS
}

\author{
LEARNING FACTORY IN INDUSTRY 4.0: BUSINESS MODEL PROPOSAL \\ Lorenna Fernandes Leal'; ${ }^{1}$ Mariana Soares de Oliveira ${ }^{2}$; Daniel Krás Borges da Silveira ${ }^{3}$; André Luiz Maciel \\ Santana ${ }^{4}$; Antônio Carlos Gomes Junior ${ }^{5}$; Diane Aparecida Reis ${ }^{6}$; André Leme Fleury ${ }^{7}$; Eduardo Zancul ${ }^{8}$
}

DOI: 10.37702/REE2236-0158.v39p157-169.2020

\begin{abstract}
RESUMO
O termo Indústria 4.0 vem ganhando força desde 2011, quando foi cunhado na Alemanha. Desde então, diversas iniciativas pedagógicas e do mercado passaram a fomentar e disseminar a necessidade de alterações na forma como a tecnologia deve ser aplicada. Diante desse contexto, esta pesquisa se propõe a estruturar um modelo de negócios que permita a concepção sustentável de uma Fábrica de Ensino - a Fábrica do Futuro -, como um espaço útil tanto para a capacitação de graduandos em engenharia e profissionais atuantes na área quanto para o desenvolvimento de atividades de pesquisa relacionadas à Indústria 4.0. Para isso, foram aplicados princípios e métodos disponíveis no Ciclo de Inovação do Startup Garage Innovation Process. Desta aplicação, foram desenvolvidas diferentes propostas de serviço na Fábrica de Ensino. Uma dessas propostas, de um workshop de qualificação em Indústria 4.0, foi testada por meio de um Produto Mínimo Viável (MVP). Os resultados indicam que a Fábrica do Futuro tem possibilidade de atender às demandas por estratégias práticas de ensino voltadas à Indústria 4.0.
\end{abstract}

Palavras-chave: Fábrica de Ensino; Indústria 4.0; Startup Garage Innovation Process; Design Thinking.

\begin{abstract}
The term Industry 4.0 has been gaining attention since 2011 when it was defined in Germany. Since then, several pedagogical and market initiatives have been fostering and disseminating the need for changes in the way technology should be applied to overcome engineering challenges. In this context, this research aims to propose a business model for the sustainable operations of a learning factory called the Factory of the Future. This learning factory should be a useful resource for training engineering undergraduates and professionals and the development of research related to Industry 4.0. To do so, we applied the principles and methods available in the Innovation Cycle from the Startup Garage Innovation Process. As a result, a service proposal for engineering professionals was developed. One of the services - a qualification workshop for Industry 4.0 - was partially tested. The results indicate that the Factory of the Future can meet the demands for novel and practical teaching strategies focused on Industry 4.0.
\end{abstract}

Keywords: Learning Factory; Industry 4.0; Startup garage innovation process; Design Thinking.

\footnotetext{
1 Mestranda em Engenharia de Produção pela Universidade de São Paulo, lorennafleal @usp.br

2 Mestranda em Engenharia de Produção pela Universidade de São Paulo, m.oliveira@usp.br

3 Mestrando em Engenharia de Produção pela Universidade de São Paulo, danielkras@gmail.com

4 Professor na Escola de Engenharia e Tecnologia da Universidade Anhembi Morumbi, Doutorando em Engenharia Elétrica (POLI) pela Universidade de São Paulo, Ims91@ usp.br

5 Professor de Processos de Fabricação e Empreendedorismo, Coordenador de Engenharia Mecânica e do Laboratório de Manufatura Avançada (4.0) no Centro Universitário FACENS, doutorando

em Engenharia Elétrica pela Universidade de São Paulo, profmateriais@yahoo.com.br

6 Doutoranda em Engenharia de Produção pela Universidade de São Paulo, diane.reis@hotmail.com

7 Professor Doutor da Universidade de São Paulo, atuando nos cursos de Engenharia de Produção (POLI) e Design (FAU), andreleme.fleury@gmail.com

8 Professor Doutor da Universidade de São Paulo (USP), atuando no curso de Engenharia de Produção (POLI), ezancul@usp.br
} 


\section{INTRODUÇÃO}

O termo Indústria 4.0, cunhado em 2011 na Alemanha, vem sendo amplamente disseminado, conceituando uma mudança de paradigma na produção industrial. De acordo com Hermann, Penterk e Otto (2016), entendese que não existe uma definição unânime para Indústria 4.0. No entanto, para este estudo, consideramos que se trata de um sistema de produção inteligente viabilizado por meio de processos de transformação e manufatura adaptáveis, otimizados, orientados para serviços interoperáveis e integráveis a tecnologias avançadas (BAYGIN et al., 2016; LU, 2017).

A fim de atender às demandas por profissionais qualificados em técnicas de produção inteligente, faz-se necessário fomentar o ensino de temas relacionados à Indústria 4.0, tanto na grade curricular de cursos superiores quanto em cursos de atualização para profissionais já inseridos no mercado de trabalho (BAYGIN et al., 2016; BENEŠOVÁ; TUPA, 2017). No contexto da Indústria 4.0, a atualização do engenheiro deve considerar o desenvolvimento de habilidades que permitam aos profissionais assumirem posições e atividades estratégicas, gerenciais, criativas, inovadoras e comunicativas (EROL et al., 2016; HECKLAU et al., 2016).

O esperado crescimento da automatização de funções operacionais pode resultar em um maior acúmulo de responsabilidade para os profissionais atuantes (HECKLAU et al., 2016). O grupo de trabalho do governo alemão em Indústria 4.0 abordou também capacitação e treinamento, enfatizando que parcerias entre universidades e empresas serão ainda mais importantes nos próximos anos (KAGERMANN; HELBIG; WAHLSTER, 2013).

Zancul et al. (2018) sugerem que o uso de metodologias ativas e experimentação prática favorecem o engajamento de alunos. Diante das demandas por estratégias de ensino mais arrojadas, surge a Fábrica de Ensino (Learning Factory), infraestrutura promissora de aprendizagem, referenciando um ambiente interdisciplinar de estudos aplicados, fortemente relacionado à indústria.

Atualmente, entende-se a Fábrica de Ensino como um ambiente controlado, com operação inspirada em uma cadeia de valor real, e que promove conhecimentos formais $\mathrm{e}$ informais, inserida em um projeto pedagógico, através de uma estrutura customizável e baseada em processos organizacionais e de fabricação de produtos (ABELE et al., 2015). Dessa forma, indústrias podem firmar parcerias com fábricas de ensino para elaborar planos de capacitação de seus funcionários, bem como para desenvolvimento e pesquisa de novas tecnologias e aplicações.

Diante desse contexto, este estudo se propõe a estruturar um modelo de negócios sustentável para uma Fábrica de Ensino focada em conceitos de Indústria 4.0, servindo tanto a propósitos acadêmicos, como graduação, pósgraduação e atividades de pesquisa, quanto ao desenvolvimento da indústria brasileira, por meio de cursos de capacitação e convênios. Para alcançar esse objetivo, foram definidos como objetivos específicos: i) aplicar os conceitos de Design Thinking e Lean Startup na concepção de um plano de negócios para uma Fábrica de Ensino; e ii) analisar os principais resultados obtidos.

Este estudo está dividido em seis seções. A primeira seção justifica e contextualiza o estudo. A segunda seção apresenta a revisão de literatura. A terceira seção detalha o método. A quarta seção evidencia os resultados, que são discutidos em seguida, na quinta seção. Por fim, a sexta seção conclui o estudo.

\section{REVISÃO DE LITERATURA}

As subseções seguintes abordam os conceitos e os princípios do Ciclo de Inovação do Startup Garage Innovation Process, conceituando Lean Startup, Startup Garage Innovation Process, Business Model Canvas e Design Thinking. Os conceitos de Fábrica de Ensino e Indústria 4.0 já foram apresentados na introdução deste estudo. 


\section{Lean Startup e Startup Garage Innovation Process}

O termo Lean Startup, cunhado por Eric Ries (EISENMANN; RIES; DILLARD, 2013), descreve organizações que seguem o modelo de empreendedorismo direcionado por hipótese. $\mathrm{Na}$ abordagem por hipóteses, os empreendedores são incentivados a trazer suas ideias para ação preparando um Produto Mínimo Viável, saindo ao mercado e coletando feedback.

O Produto Mínimo Viável (do inglês Minimum Viable Product - MVP) é aquele que compreende o menor grupo de funcionalidades e características suficientes para validar (ou não) o modelo de negócio projetado, conforme avaliações dos usuários potenciais. Esse exercício de validação do modelo de negócios, geralmente inclui as etapas: ii) compreender e elaborar uma ponto de vista para o problema; ii) elaborar propostas de solução; iii) priorizar uma proposta de solução e projetar um modelo de negócio que entregue essa solução aos usuários, especificando uma proposta de valor, meios de relacionamento com clientes e forma de remuneração do negócio; iv) especificar o MVP, considerando as características mais importantes do produto e as restrições dos usuários; v) testar o modelo de negócio e o MVP; vi) analisar os resultados dos testes; vii) a partir dos resultados obtidos nos testes, decidir entre perseverar (de modo que o valor proposto faça sentido aos usuários) ou "pivotar" (descontinuando o projeto caso a proposta de valor e/ou o modelo de negócios não seja validado) (BLANK; DORF, 2012).

As características dessa metodologia consideram: investir energia e conhecimento na experimentação, reconhecimento dos erros e ajustes de projeto; conversar e trocar informações diretamente com os usuários potenciais; e analisar o que é essencial para entregar valor (BLANK, 2013). Os ciclos iniciais de testes estruturados antecipam problemas e suas soluções. Isso é, auxiliam a revisar o modelo de negócio e evidenciar o que funciona, antes de se comprometer capital e tempo de desenvolvimento, buscando assim garantir melhor desempenho dos empreendimentos.

Uma das propostas de aprimoramento da abordagem de empreendedorismo direcionado por hipótese, que traz tanto essas etapas quanto a relação (sistêmica) entre elas, apresenta-se no Startup Garage Innovation Process (Figura 1) - modelo que contempla desde o momento inicial da ideação, da compreensão do problema, até sua formatação em um ponto de vista e perspectiva, possibilitando desenvolver uma solução para uma demanda real e, a partir disso, fabricar e testar essa solução em conjunto com os usuários potenciais. Dessa forma, o Startup Garage Innovation Process permite avaliação da percepção dos usuários potenciais sobre o valor (ou não) que o produto (ou serviço) entrega, garantindo a validação do modelo de negócio.

Figura 1 - Representação do Startup Garage Innovation Process

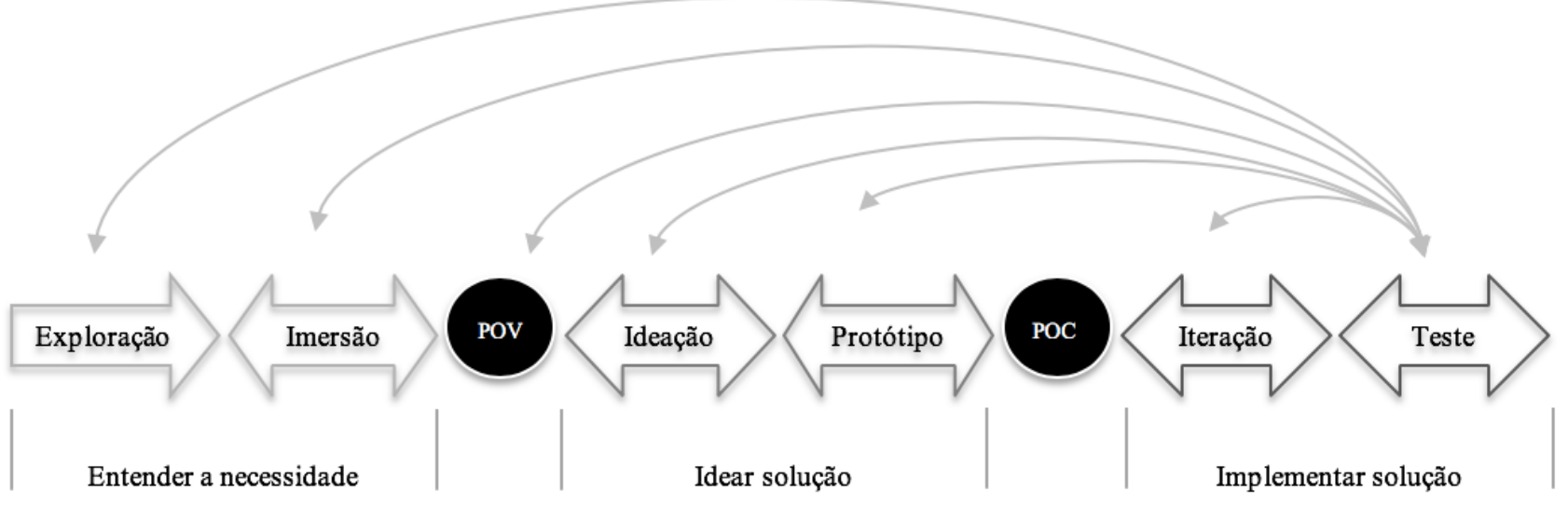

Fonte: Adaptado de Zenios (2016). 


\section{Business Model Canvas (BMC)}

O Business Model Canvas, proposto por Osterwalder e Pigneur (2010), pode ser definido como uma ferramenta para auxiliar no entendimento e definição de um modelo de negócios. Segundo os autores, um modelo de negócios visa a descrever como uma organização pode capturar, criar e entregar valor. O BMC se apresenta de maneira simples e visual, permitindo a criação de um modelo de negócios simplificado em apenas uma página, dividido em nove componentes que refletem a estratégia organizacional (Figura 2). Dessa forma, o BMC procura dar destaque ao que é desenvolvido para se elevar o valor entregue aos consumidores, guiando a gestão da organização na busca pela sustentabilidade (TRIMI; BERBEGAL-MIRABENT, 2012).

Figura 2 - Representação do Business Model Canvas

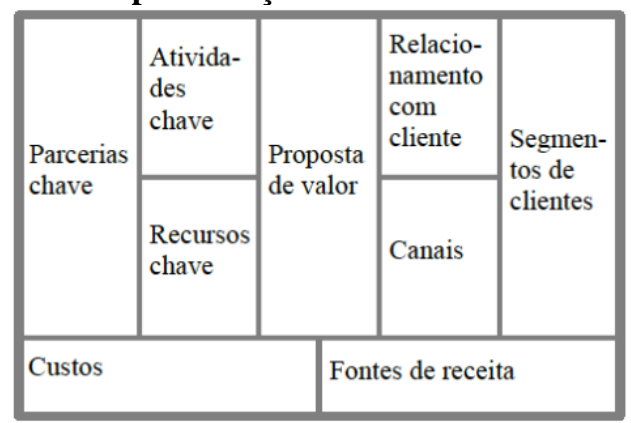

Fonte: Adaptado de Osterwalder e Pigneur (2010).

\section{Design Thinking (DT)}

Popularizado por Brown (2008), o Design Thinking pode ser definido como abordagem para a solução de problemas que se utiliza da sensibilidade dos designers e de seus métodos para identificar as necessidades dos usuários e transformá-las em oportunidade de negócio. Segundo Fleury, Stabile e Carvalho (2016), o DT emergiu como uma abordagem que permite a promoção de inovações radicais e incrementais em empresas que busquem por serviços e produtos inovadores. Seus princípios básicos são: empatia - colocar as pessoas em primeiro lugar; cooperação interdisciplinaridade para a solução de problemas complexos; pensamento integrativo - utilizar tanto pensamento analítico quanto intuitivo; e experimentação - explorar o problema e suas limitações de maneira criativa, com uso frequente de protótipos rápidos (BROWN, 2008; TSCHIMMEL, 2012).

O DT não deve ser interpretado como uma sequência ordenada de etapas, mas sim como um sistema de sobreposição de espaços (BROWN; WYATT, 2010). De maneira geral, esses espaços de sobreposição (Figura 3) podem ser: inspiração - buscar o entendimento do problema e a empatia com os usuários finais; ideação - gerar ideias para solução do problema, testes e melhoria destas; implementação - transformar a ideia final em um plano de ação para sua implementação, geralmente por meio do uso de protótipos (BROWN, 2008; TSCHIMMEL, 2012).

Figura 3 - Representação visual dos três espaços de sobreposição do Design Thinking

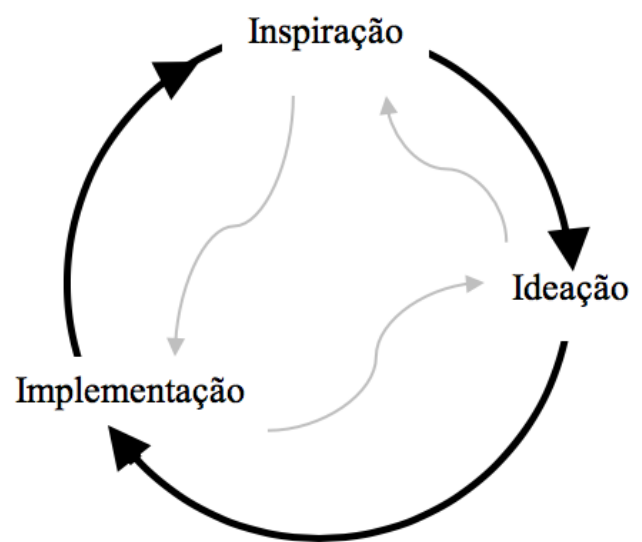

Fonte: Adaptado de Brown (2008).

Algumas ferramentas são utilizadas em cada um desses espaços, tais como as apresentadas na Tabela 1 (TSCHIMMEL, 2012; D. SCHOOL, 2010).

Tabela 1 - Ferramentas utilizadas na aplicação do Design Thinking

\begin{tabular}{|l|l|}
\hline Ferramenta & Descrição \\
\hline Entrevistas & $\begin{array}{l}\text { Captação as necessidades e ponto } \\
\text { de vista dos usuários. }\end{array}$ \\
\hline Persona & $\begin{array}{l}\text { Personagem fictício que permita a } \\
\text { criação de empatia com os usuários } \\
\text { e suas experiências. }\end{array}$ \\
\hline $\begin{array}{l}\text { Jornada do } \\
\text { usuário }\end{array}$ & $\begin{array}{l}\text { Representação gráfica de cada etapa } \\
\text { pela qual o usuário passa ao utilizar } \\
\text { um serviço ou produto. }\end{array}$ \\
\hline $\begin{array}{l}\text { Point of View } \\
\text { (POV) }\end{array}$ & $\begin{array}{l}\text { Síntese do ponto de vista e insights } \\
\text { do grupo sobre o problema, }\end{array}$ \\
\hline
\end{tabular}




\begin{tabular}{|l|l|}
\hline & $\begin{array}{l}\text { usualmente apresentando o seguinte } \\
\text { formato: "Usuário" precisa de } \\
\text { "necessidade" porque "insight". }\end{array}$ \\
\hline Brainstorming & $\begin{array}{l}\text { Geração de muitas ideias, em grupo, } \\
\text { para um problema específico. }\end{array}$ \\
\hline
\end{tabular}

Fonte: elaborada pelos autores.

\section{METODOLOGIA}

Este estudo consiste em uma pesquisaação, que propõe soluções para problemas relevantes e reais que são enfrentados no contexto em que os autores estão inseridos; estes participam de maneira colaborativa, sendo membros ativos do contexto estudado (COUGHLAN; COUGHLAN, 2002).

A pesquisa-ação foi desenvolvida com base em um projeto no contexto da educação, denominado Fábrica do Futuro, e recebeu o rigor de uma pesquisa científica, ideia defendida por Biancolino et al. (2012). A pesquisa é fundamentada em referências bibliográficas para a conceituação das variáveis de interesse, conforme o apresentado no capítulo de revisão de literatura.

Detalhando-se o cenário da pesquisa-ação, a Fábrica do Futuro (Figura 4) segue os moldes de uma Fábrica de Ensino, sendo estruturada com o propósito de apresentar tecnologias avançadas de Indústria 4.0 por meio de uma linha de produção, que seja didática e ao mesmo tempo permita a experiência próxima de se vivenciar uma fábrica real.

\section{Figura 4 - Estrutura da Fábrica do Futuro}

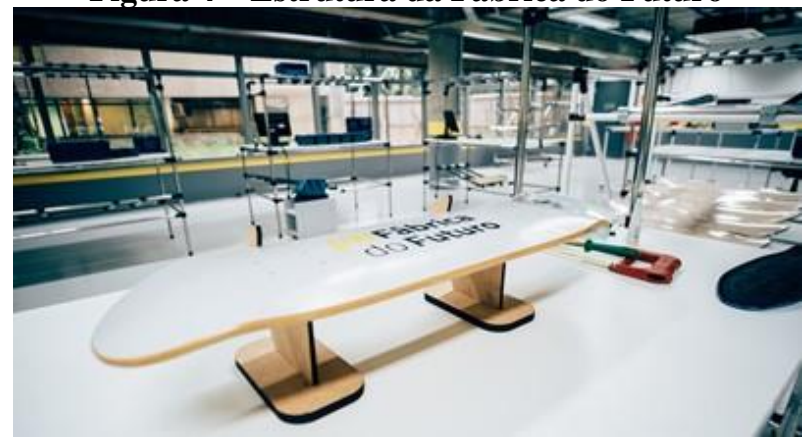

Fonte: acervo dos autores.

Com base nos recursos e estrutura disponíveis na Fábrica do Futuro, foram especificados previamente a este trabalho um único produto base - um skate - e toda a sua linha de montagem. $\mathrm{O}$ processo foi estruturado de forma a suportar um projeto pedagógico, abordando conceitos de customização em massa, gêmeo digital, manufatura aditiva, integração de sistemas, visão computacional, single-board computers, Manufacturing Execution System (MES) e Enterprise Resource Planning (ERP). Outras aplicações de tecnologias estão ainda em desenvolvimento e planeja-se garantir sempre a atualização das demonstrações, alinhando ao estado da arte em tecnologia industrial.

\section{RESULTADOS}

Nesta seção são apresentados os resultados da aplicação do Startup Garage Innovation Process, complementado pelo Business Model Canvas e pelo Design Thinking, para a criação do modelo de negócios para a Fábrica de Ensino anteriormente citada. Inicialmente são descritos os resultados obtidos pelas entrevistas, que foram construídas com o intuito de explorar as necessidades dos clientes potenciais. Em sequência, foram definidas as personas, que representam o comportamento dos principais clientes potenciais. Na terceira etapa, foi apresentada a jornada do usuário, viabilizando um brainstorm direcionado à implementação de um primeiro protótipo, que gerou um modelo de negócio e, por fim, a implementação do Produto Mínimo Viável.

\section{Entrevistas}

A primeira etapa do Startup Garage Innovation Process, Exploração e Imersão, inicia-se por meio do entendimento do problema e do usuário. O Design Thinking e suas ferramentas possuem grande utilidade nessa etapa. Dessa forma, foram realizadas entrevistas com usuários potenciais da Fábrica do Futuro, abordando dois grupos: grupo A, formado por integrantes de startups e gestores de pequenas e médias empresas; e grupo B, composto por estudantes de engenharia, graduandos e pós-graduandos.

As sete entrevistas do grupo A abrangeram questões sobre prévia utilização de manufatura aditiva para prototipagem e personalização de 
produtos para seus clientes, bem como o interesse e desejo de se manter atualizado no tema Indústria 4.0. Identificou-se que o desejo de implementação existe, mas que os profissionais não compreendem bem o que é Indústria 4.0 e possuem a visão de que a implementação dessas tecnologias pode ser muito custosa. Para esse público, a expectativa é ofertar treinamentos acerca dos conceitos de Indústria 4.0, bem como demonstrar a aplicação de tecnologias, buscando quebrar o paradigma de que é custoso, permitindo a transferência de conhecimento para a indústria.

Relativamente ao grupo $\mathrm{B}$, foram entrevistados 11 estudantes do curso de Engenharia de Produção de uma universidade do estado de São Paulo. Desse grupo, nove estudantes estão no quinto ano de graduação, um no terceiro ano e um no quarto ano. A entrevista foi estruturada, utilizando-se de perguntas que incentivassem comentários adicionais. Os resultados indicaram que o conceito de Indústria 4.0 é nebuloso e que os entrevistados não possuem plena ciência de seu significado. Para esse público, a expectativa é ofertar disciplinas específicas de aplicação, desenvolvendo projetos reais na Fábrica ou em conjunto com empresas, além de efetivar visitas para demonstração de tecnologias da Indústria 4.0 na Fábrica.

Os resultados das entrevistas inspiraram a criação de quatro personas.

\section{Personas e Point of View (POV)}

As personas definidas são: estudante de graduação; pesquisador ou estudante de pósgraduação; startup; e gestor de equipes de produção. Cada persona representa o perfil de um usuário potencial da Fábrica do Futuro.

Devido ao maior potencial de promover a sustentabilidade da Fábrica do Futuro por meio de cursos de capacitação e atualização, do desenvolvimento de projetos em parceria com a indústria e, consequentemente, da atração das demais personas apresentadas, a persona do gestor de equipes de produção foi selecionada inicialmente. Sua descrição detalhada pode ser vista na Tabela 2.
Tabela 2 - Persona do gerente de equipes de produção

\begin{tabular}{|l|l|}
\hline Motivações & $\begin{array}{l}\text { Particularidades } \\
\text { (histórias) }\end{array}$ \\
\hline $\begin{array}{l}\text { Corporativo - elevar o } \\
\text { nível de qualificação da } \\
\text { equipe e melhorar a } \\
\text { entrega da produção; } \\
\begin{array}{l}\text { Pessoal - manutenção } \\
\text { da empregabilidade. }\end{array}\end{array}$ & $\begin{array}{l}\text { Faz dez anos que não } \\
\text { busca nenhum curso } \\
\text { imersivo para } \\
\text { desenvolvimento de } \\
\text { novas competências. }\end{array}$ \\
\hline Objetivos & Comportamentos \\
\hline $\begin{array}{l}\text { Conhecer tecnologias } \\
\text { que possam ser um } \\
\text { diferencial na } \\
\text { produção. }\end{array}$ & $\begin{array}{l}\text { Costuma descrever } \\
\text { mudanças pelos impactos } \\
\text { negativos no conforto da } \\
\text { produção. } \\
\text { Busca melhorias com } \\
\text { base na compra de novos } \\
\text { equipamentos. } \\
\text { Tem insegurança sobre } \\
\text { mudanças recentes em } \\
\text { relação no contexto de } \\
\text { negócios. }\end{array}$ \\
\hline
\end{tabular}

Fonte: elaborada pelos autores.

O POV definido para essa persona é: "O gerente precisa entender sobre Indústria 4.0 para manter a sua empregabilidade e melhorar os resultados de sua empresa". A partir do POV, foi elaborada a jornada do usuário.

\section{Jornada do usuário}

A jornada do usuário (Figura 5) se inicia pelo contato com conceitos da Indústria 4.0, quando o usuário se interessa por entender melhor essa tendência e aprofundar seus conhecimentos, realizando pesquisas na internet e perguntando a pessoas próximas. Essa etapa foi nomeada como "coleta de informações". Em seguida, o usuário percebe a necessidade de conhecimento mais profundo sobre a evolução tecnológica ("autorreflexão") e, então, busca se atualizar para manter a empregabilidade quando a indústria adotar as tecnologias avançadas. O usuário então realiza o "contato inicial" com a Fábrica do Futuro, pessoalmente, por meio de telefone ou e-mail, e se inscreve para um dos cursos/serviços. 
Figura 5: Representação da jornada do usuário

\section{Coleta de informacões}

Autorreflexão

\section{Contato inicial}

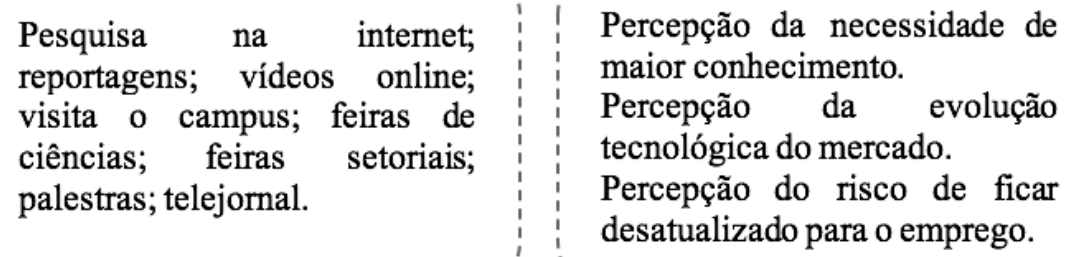

Percepção da necessidade de maior conhecimento.

Percepção da evolução tecnológica do mercado.

Percepção do risco de ficar desatualizado para o emprego.
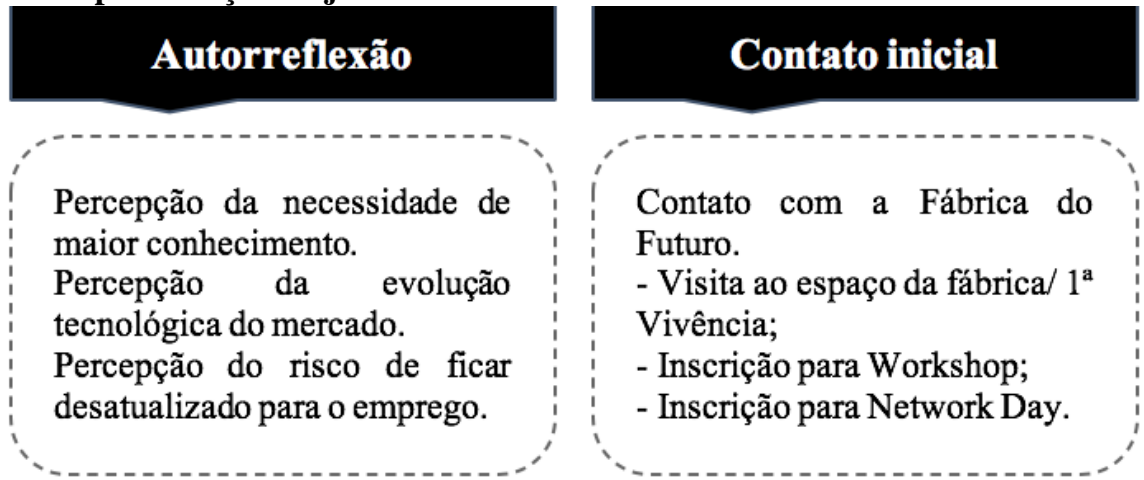

Fonte: elaborada pelos autores.

Com base nas informações e insights coletados, foi realizado um brainstorm para geração de ideias e definição do primeiro protótipo.

\section{Brainstorm e Protótipo}

$\mathrm{O}$ brainstorming, realizado com a participação dos autores, foi registrado por meio de mapa mental descrito na Figura 6.

Como resultado do brainstorming, foram elaboradas três opções de serviço: i) Serviço A - visita guiada pela infraestrutura da Fábrica do Futuro e rápida introdução aos conceitos de Indústria 4.0, serviço que seria gratuito, com o objetivo de ambientar os interessados e apresentá-los às duas outras opções; ii) Serviço B - workshop combinando conhecimento teórico e prático, incluindo a reflexão sobre como aplicar as tecnologias; Serviço $\mathrm{C}$ - evento de networking no qual empresas interessadas em absorver as tecnologias da Indústria 4.0 encontram os fornecedores das tecnologias.
Figura 6 - Resultados do Brainstorm

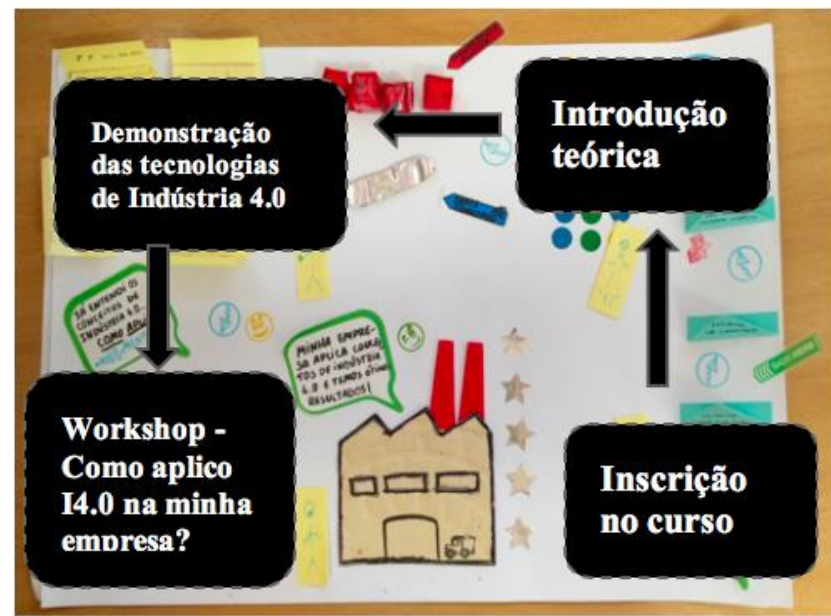

Fonte: elaborada pelos autores.

O protótipo realizado apresenta a jornada do usuário para o Serviço B, considerado como o mais complexo e, possivelmente, o que pode agregar mais valor ao usuário. $\mathrm{O}$ serviço apresenta uma introdução teórica à Indústria 4.0, demonstrando suas tecnologias, e é finalizado com um workshop em grupo, no qual o participante reflete e propõe um roadmap de como implementar as tecnologias de Indústria 4.0 em sua empresa (Figura 7). 
Figura 7 - Primeiro protótipo do Serviço B

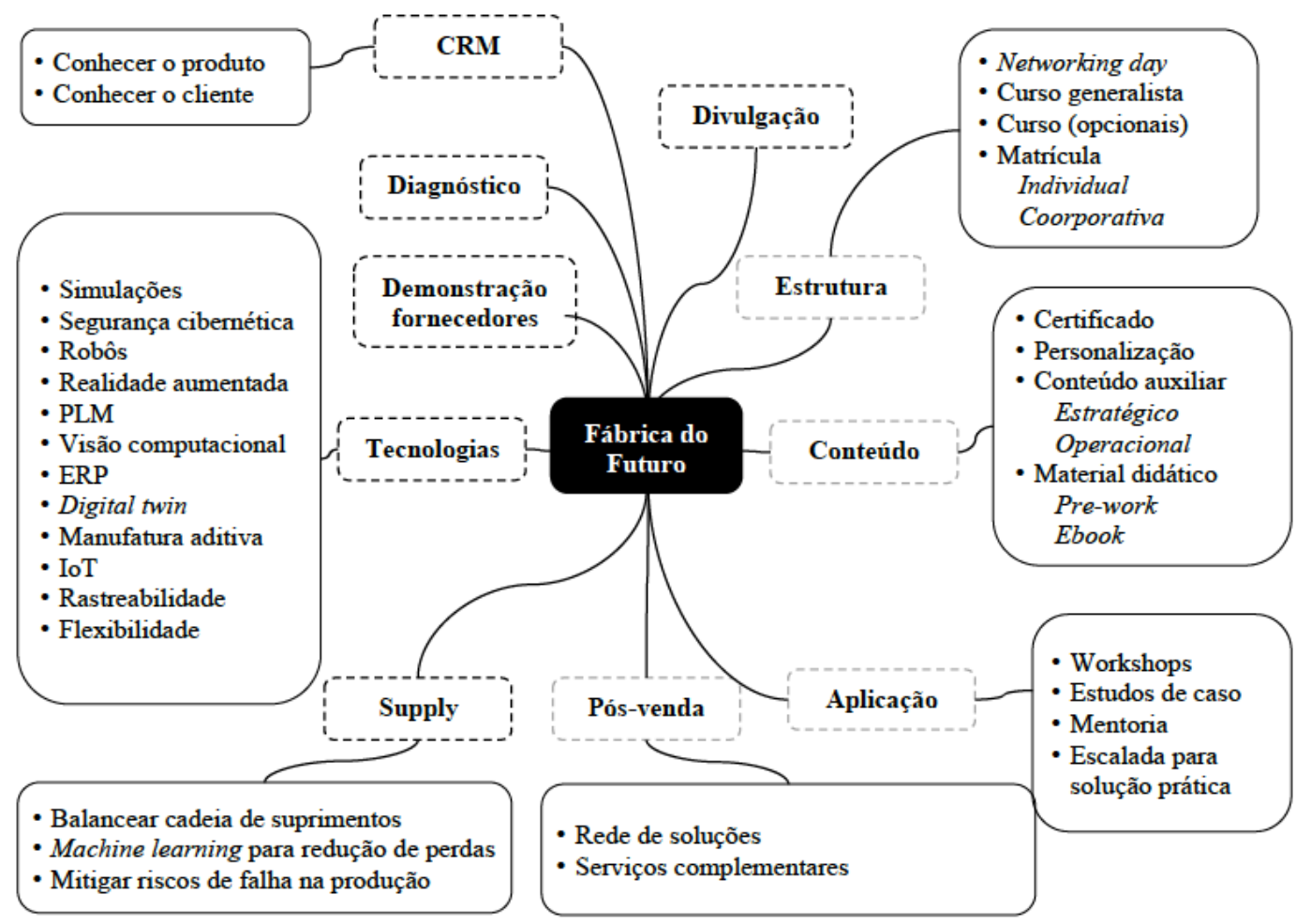

Fonte: elaborada pelos autores.

Com o desenvolvimento do primeiro protótipo, foi aplicado o Business Model Canvas (BMC) para detalhamento das possibilidades do nos Serviços B.

\section{Business Model Canvas (BMC)}

Com base no protótipo exibido na Figura 7, foi elaborado o Business Model Canvas (Figura 8) para auxiliar as etapas posteriores deste trabalho. Na Figura 8, os post-its representam as dimensões de negócio relacionadas aos potenciais clientes do Serviço B.

Figura 8 - Business Model Canvas aplicado

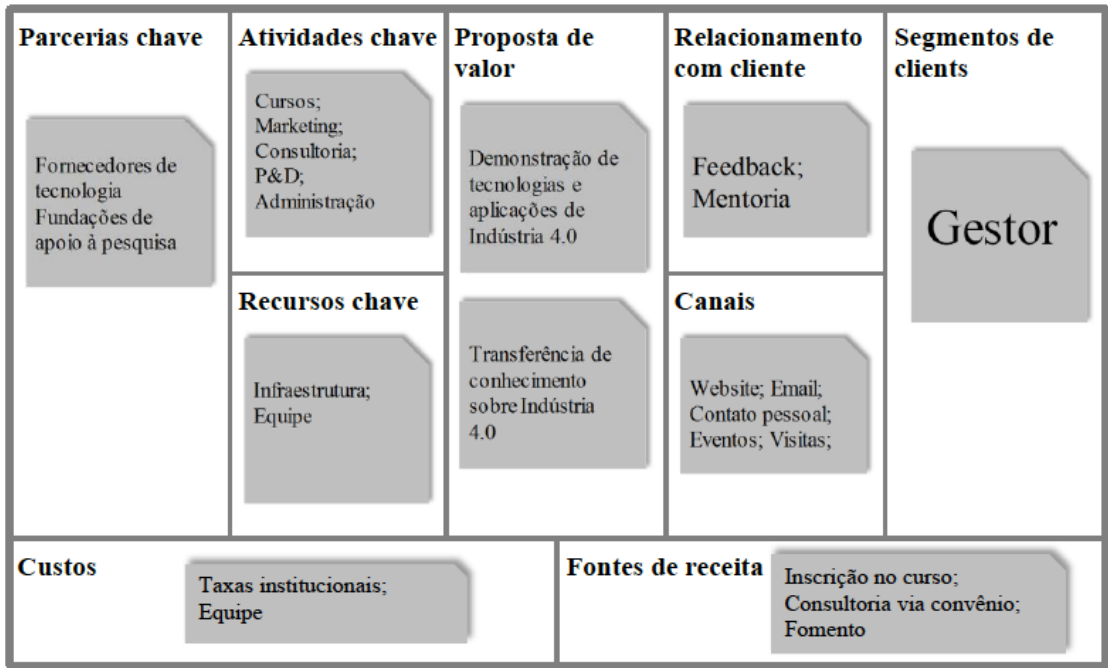

Fonte: elaborada pelos autores. 


\section{Produto Viável Mínimo (MVP)}

Buscando-se verificar se o serviço escolhido possui valor para os usuários potencias, foram definidas duas abordagens para testes: i) a criação de uma landing page para a divulgação do curso (Figura 9) e ii) uma visita guiada à fábrica para apresentação das tecnologias de Indústria 4.0 presentes (Figura $10)$.

Figura 9 - Representação da landing page
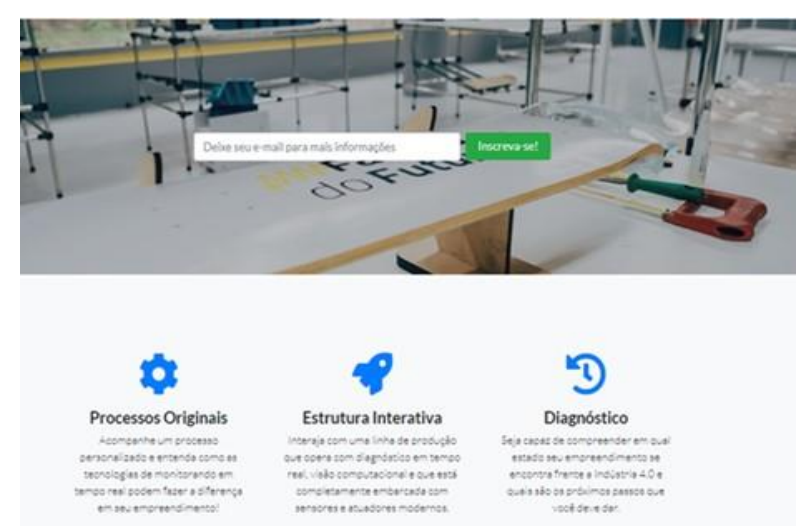

Fonte: acervo dos autores.

Figura 10 - Visita guiada à Fábrica do Futuro para apresentação das tecnologias de Indústria 4.0

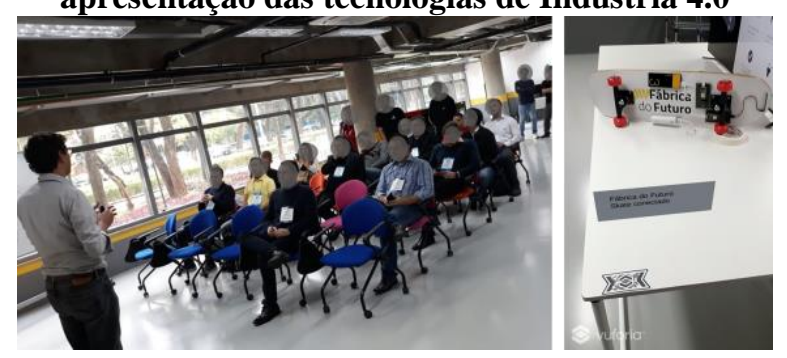

Fonte: acervo dos autores.

A landing page construída no primeiro MVP apresentou algumas informações aos usuários com o intuito de motivá-los a uma inscrição prévia em um possível curso de atualização à Indústria 4.0. A landing page resultou em nove respostas ao longo de dois dias de publicação em redes sociais como Linkedin e Facebook.

Já a visita à Fábrica buscou identificar o interesse de possíveis clientes, com perfil similar ao da persona escolhida, em relação à fábrica e suas tecnologias demonstradas. A apresentação das tecnologias de Indústria 4.0 foi realizada para dois grupos de aproximadamente 25 pessoas e foram identificados como maiores pontos de interesse as aplicações de tecnologia como visão computacional integrada ao Manufacturing Execution System (MES) e um protótipo de um smart product conectado com base em tecnologias de Internet das Coisas.

\section{DISCUSSÃO}

A partir de pesquisas teóricas sobre as competências necessárias para um profissional na $4^{\mathrm{a}}$ Revolução Industrial e definindo as Fábricas de Ensino como potenciais espaços para desenvolvimento dessas competências, o artigo se propôs a estabelecer e validar um modelo de negócio sustentável para uma Fábrica do Futuro, utilizando-se da abordagem do Startup Garage Innovation Process.

Após a seleção e aplicação das ferramentas da abordagem (entrevistas, personas, point of view, jornada do usuário, brainstorm, protótipo, BMC e MVP) ao objeto de estudo, foram obtidos indicadores satisfatórios quanto ao interesse de usuários potenciais da Fábrica do Futuro. Entretanto, foi realizado apenas um ciclo de prototipagem e teste do modelo por meio de MVPs. A abordagem recomenda que esses ciclos sejam conduzidos de forma iterativa, permitindo que a equipe aprenda com os resultados de cada ciclo e aprimore o protótipo conforme as reações dos clientes potenciais. Como aprendizado desse primeiro ciclo, foi identificada a necessidade de detalhamento da jornada do usuário, especialmente na coleta de informações; como consequência, percebe-se ainda a oportunidade para maior investimento em estratégias de marketing para a Fábrica do Futuro em meios digitais, aumentando o alcance da divulgação. As necessidades identificadas serão consideradas nos próximos ciclos de iteração visando ao aprimoramento da solução.

A proposta de serviço B (workshop que combina conhecimento teórico e prático, incluindo a reflexão sobre como aplicar as tecnologias) contempla competências relacionadas à autonomia, à sociabilidade, à iniciativa e, ainda, competências técnicas por meio de: i) workshop em grupo para proposição 
de roadmap de implementação de tecnologias avançadas em sua empresa e ii) introdução à Indústria 4.0, demonstração de suas tecnologias e conexão entre fornecedores e clientes.

A proposta foi avaliada por meio de visita à Fábrica do Futuro, evidenciando que os participantes responderam de forma positiva; porém, uma vez que o público tinha um caráter bem técnico, é possível que este já possuísse conhecimentos prévios em Indústria 4.0. Essa experiência levantou a dúvida sobre a existência ou não de uma demanda de conhecimentos teóricos sobre a Indústria 4.0, ou se uma abordagem de aplicação de tecnologia teria mais valor para esse tipo de usuário. Contudo, faz-se necessária a aplicação de novos testes para mitigar tais dúvidas, já que essa foi ainda a primeira iteração no ciclo de prototipagem e teste.

\section{CONSIDERAÇÕES FINAIS}

Em suma, tem-se o desenho de um modelo de negócio sustentável de uma Fábrica de Ensino por meio da Fábrica do Futuro, que atende às demandas por estratégias de Ensino de Engenharia arrojadas, com experiências práticas e alinhadas à Indústria 4.0. Enxerga-se potencial na continuidade deste estudo. Como próximos passos serão realizados ciclos iterados de prototipagem e teste, atendendo às necessidades das quatro personas levantadas neste artigo.

Em estudos futuros, cabe utilizar do passoa-passo aqui apresentado para a construção de novos modelos de negócios e a realização de mais ciclos de desenvolvimento de abordagens de ensino para a Fábrica do Futuro.

\section{AGRADECIMENTOS}

Agradecemos o apoio recebido da ABDI (Agência Brasileira de Desenvolvimento Industrial) e do Fundo Patrimonial Amigos da Poli para a implantação da Fábrica do Futuro.

\section{REFERÊNCIAS}

ABELE, E. et al. Learning factories for research, education, and training. Procedia CiRp, v. 32, p. 1-6, 2015.

BAYGIN, M. et al. An effect analysis of industry 4.0 to higher education. 15th

International Conference on Information Technology Based Higher Education and Training, p.1-4, 2016.

BENEŠOVÁ, A.; TUPA, J. Requirements for education and qualification of people in Industry 4.0. Procedia Manufacturing, v. 11, p. 2195-2202, 2017.

BIANCOLINO, C. A. et al. Protocolo para elaboração de relatos de produção técnica. Revista de Gestão e Projetos, São Paulo, v. 3, n. 2, p. 294-307, mai./ago. 2012.

BLANK, S. Why the lean start-up changes everything. Harvard Business Review, $p$ 1-9, May, 2013.

BLANK, S.; DORF, B. The Startup Owner's Manual. Pescadero, CA: K\&S Ranch. 2012.

BROWN, T. Design thinking. Harvard Business Review, v. 86, n. 6, p. 84, 2008.

BROWN, T.; WYATT, J. Design thinking for social innovation. Development Outreach, v. 12, n. 1, p. 29-43, 2010.

COUGHLAN, P.; COUGHLAN, D. Action research for operations management. International Journal of Operations \& Production Management, v. 22, n. 2, p. 220-240, 2002.

D.SCHOOL. Bootcamp bootleg. Institute of Design at Stanford, 2010.

EISENMANN, T.; RIES, E.; DILLARD, S. Hypothesis-driven entrepreneurship: The lean startup. Harvard Business School, p. $1-26,2013$. 
EROL, S. et al. Tangible Industry 4.0: A scenario-based approach to learning for the future of production. Procedia CIRP, v. 54, p. 13-18, 2016.

FLEURY, A. L.; STABILE, H.; CARVALHO, M. M. An overview of the literature on design thinking: Trends and contributions. The International Journal of Engineering Education, v. 32, n. 4, p. 1704-1718, 2016.

HECKLAU, F. et al. Holistic approach for human resource management in Industry 4.0. Procedia CIRP, v. 54, p. 1-6, 2016.

HERMANN, M.; PENTEK, T.; OTTO, B. Design principles for industrie 4.0 scenarios. 49th Hawaii international conference on system sciences (HICSS), p. 3928-3937, 2016.

KAGERMANN, H; HELBIG, J; WAHLSTER, W. Recommendations for implementing the strategic initiative Industrie 4.0: Securing the future of German manufacturing industry; final report of the Industrie 4.0 Working Group. Forschungsunion, 2013.
LU, Y. Industry 4.0: A survey on technologies, applications and open research issues. Journal of Industrial Information Integration, v. 6, p. 1-10, 2017.

OSTERWALDER, A.; PIGNEUR, Y. Business model generation: A handbook for visionaries, game changers, and challengers. John Wiley \& Sons, 2010.

TRIMI, S.; BERBEGAL-MIRABENT, J. Business model innovation in entrepreneurship. USA: Springer, $p$. 449-465, 2012.

TSCHIMMEL, K. Design thinking as an effective toolkit for innovation. ISPIM Conference Proceedings, 2012.

ZANCUL, E. et al. O cenário atual da disciplina de Introdução à Engenharia no Brasil: Uma oportunidade a ser aproveitada. Research and Innovation in Brazilian Education, 2018.

ZENIOS, S. Critical Questions When Lauching Innovation Is the Team Right? Is it Time to Shift Gears? IESEinsight, Issue 30, third quarter 2016.

\section{DADOS BIOGRÁFICOS DOS AUTORES}

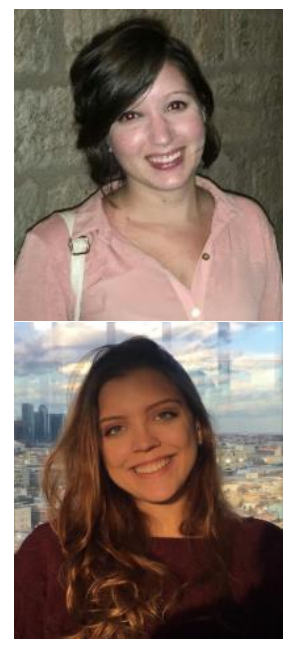

Lorenna Fernandes Leal é graduada em Engenharia de Produção pela Universidade do Estado do Rio de Janeiro (2018) e doutoranda em Engenharia de Produção pela Universidade de São Paulo.

Mariana Soares de Oliveira é graduada em Engenharia Mecânica pela Universidade Federal da Paraíba (2018) e mestranda em Engenharia de Produção pela Universidade de São Paulo. 

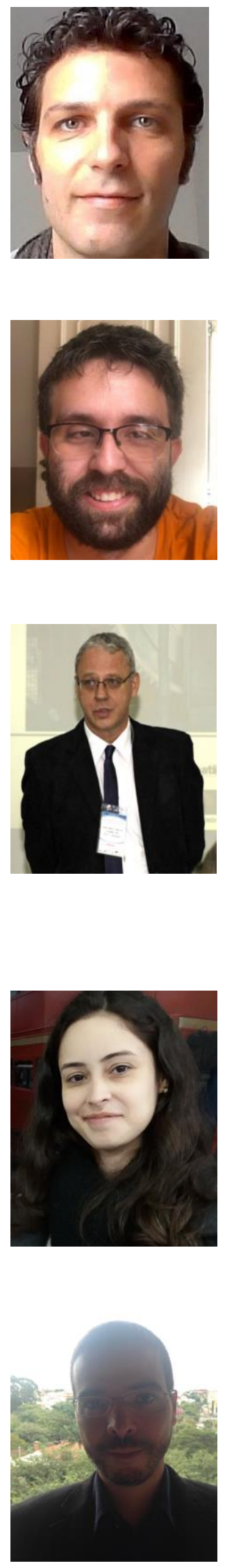

Daniel Krás Borges da Silveira é gerente do FabLab no Insper - Instituto de Ensino e Pesquisa e mestrando em Engenharia de Produção pela Universidade de São Paulo. Formado em Filosofia pela Universidade Federal do Rio Grande do Sul (2000), em Engenharia de Controle e Automação pela Pontifícia Universidade Católica do Rio Grande do Sul (2007) e no FabAcademy (2017).

André Luiz Maciel Santana é bacharel em Engenharia Industrial Mecânica (2013) e Mestre em Computação Aplicada (2015) pela Universidade do Vale de Itajaí. Iniciou seu doutorado no Programa de Pós-Graduação em Engenharia Elétrica na Escola Politécnica da Universidade de São Paulo em 2018. Atualmente é professor na Escola de Engenharia e Tecnologia da Universidade Anhembi Morumbi e atua nas linhas de pesquisa de Ensino de Engenharia e Computação, STEAM, Ciência de Dados e Internet das Coisas. Foi finalista do prêmio Santander Empreendedorismo em 2012, vencedor da edição do Sinapse da Inovação em 2017 e é Fellow do MIT - Lemann Creative Learning desde 2015.

Antonio Carlos Gomes Junior é graduado em Eletromecânica pela ETE Getúlio Vargas SP (1982), em Engenharia Metalúrgica pela Faculdade de Engenharia Industrial - FEI (1988), MBA em Gestão de Negócios pela Fundação Getúlio Vargas (2002), Mestre em Processos Industriais pelo Instituto de Pesquisas Tecnológicas de São Paulo IPT (2013), tem Especialização em Liderança e Gestão pela Wirtschaftsuniversität Wien (2014) e é doutorando em Engenharia Elétrica pela Universidade de São Paulo com ênfase em Educação. Professor no Centro Universitário FACENS nas áreas de Processos de Fabricação e Empreendedorismo. Foi coordenador de P\&D, gestor de relações internacionais, núcleos de competição e FabLab. Atualmente é coordenador de Engenharia Mecânica, Laboratório de Manufatura Avançada (4.0) e Assessor da Diretoria FACENS.

Diane Aparecida Reis é graduada em Administração pela Universidade Presbiteriana Mackenzie, especialista em Administração de Serviços pela Fundação Vanzolini, mestre em Engenharia de Produção pela Universidade de São Paulo e doutoranda em Engenharia de Produção pela Universidade de São Paulo. Tem experiência nas áreas de Planejamento Financeiro, Logística e Atendimento ao Consumidor e em organizações como: NEC do Brasil, Banco Santander, C\&A Modas e B2W Digital. Desenvolve pesquisa nas áreas de ensino do empreendedorismo, desenvolvimento de competências e intenção empreendedora, Design Thinking, Lean Startup e Business Model Canvas.

André Leme Fleury é graduado em Engenharia Mecânica de Produção pela Universidade de São Paulo (1995), mestre em Engenharia de Produção pela Universidade Federal de Santa Catarina (2000) e doutor em Engenharia de Produção pela Universidade de São Paulo (2007), com período sanduíche na Universidade de Cambridge (2004-2005). Professor Doutor da Universidade de São Paulo, atuando nos cursos de Engenharia de Produção (POLI) e Design (FAU). Desenvolve pesquisas nas áreas de desenvolvimento de produtos, serviços e empreendimentos e gestão de tecnologias nos temas de Technology Roadmapping, Design Thinking, Lean Startup e serviços tecnológicos. 


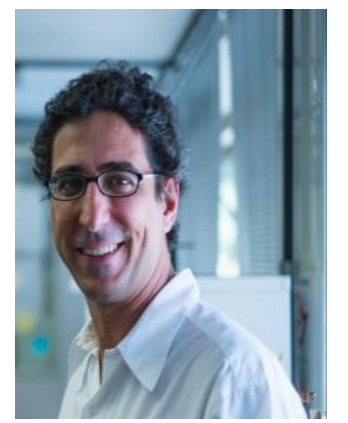

Eduardo Zancul é Professor Doutor do Departamento de Engenharia de Produção da Escola Politécnica da Universidade de São Paulo (POLI-USP). Formado em Engenharia Mecânica (1997) e tem mestrado (2000) e doutorado (2009) em Engenharia de Produção, todos pela Universidade de São Paulo. Foi pesquisador na RWTH Aachen University, na Alemanha, entre 2005 e 2007, e visiting scholar na Stanford University nos EUA durante três meses em 2015. Na POLI-USP foi um dos fundadores e é um dos coordenadores do InovaLab@POLI. Suas áreas de pesquisa incluem Métodos de Desenvolvimento de Produtos, Manufatura Avançada e Ensino de Engenharia, com foco em Ensino de Projetos. 